\title{
Multinodular and Vacuolating Neuronal Tumor in the Brain Depth - Atypical Presentation of a New Tumor: A Case Report
}

\author{
Narvaez EO ${ }^{1}$, Marussi VHR ${ }^{1}$, Junior CLS ${ }^{1}$, Hatano $\mathrm{NT}^{1}$, Maluf FC ${ }^{2}$ and Amaral LLF ${ }^{1}$ \\ ${ }^{1}$ Department of Neuroradiology, BP Medicina Diagnóstica, Hospital Beneficência Portuguesa de São Paulo - São Paulo - SP, Brazil \\ ${ }^{2}$ Department of Oncology, Hospital Beneficência Portuguesa de São Paulo, São Paulo - SP, Brazil
}

\begin{abstract}
Introduction: Multinodular and Vacuolating Neuronal Tumors (MVNTs) are clinically benign lesions and was recently included in the last World Health Organization Classification of Tumors of the Central Nervous System (WHO - 2016). Recent studies have discovered specific immunomarkers that classifies the MVNTs as a new neoplasm group placed within the section covering "Neuronal and mixed neuronal-glial tumors" as a WHO grade I lesion.

Case report: We present a case of a probable MVNT in a 55-year old woman, who presented with chronic pattern seizures. Conventional MRI revealed an infiltrative and multinodular pattern lesion localized in the deep left cerebral hemisphere compromising the ventral posteromedial and pulvinar surfaces of thalamus, lateral geniculate body, choroidal fissure, mammillothalamic tract, fornix and septum pellucidum, anterior commissure, extending to contralateral globus pallidus and internal capsule. Dynamic susceptibility contrast-enhanced MR perfusion and Arterial Spin Labeling (ASL) technique didn't show any abnormalities. MR spectroscopy demonstrated a mild increase in Choline (Cho) and mild reduction in $\mathrm{N}$-acetyl aspartate (NAA). No substantial difference both clinically and radiologically was observed on five years follow up.

Conclusion: The diagnosis of MVNT was made considering the extremally benign biological behavior of this tumor and the typical imaging features, despite of deep localization of the lesion, and once this kind of lesion was previously described as a "don't touch lesion" the histological, biopsy was not performed.
\end{abstract}

Keywords: Multinodular and vacuolating neuronal tumors $\cdot$ Neuronal and mixed neuronal-glial tumors $•$ WHO classification of brain tumors $\bullet$ Brain tumors immunomarkers $\cdot \mathrm{MRI} \cdot \mathrm{ASL}$ perfusion imaging

\section{Introduction}

Brain Multinodular and vacuolating neuronal tumor (MVNT) is an entity that was first described by Huse et al. in 2013 as a benign seizureassociated lesion with a characteristic histopathologic appearance [1]. MVNT was included in the last World Health Organization Classification of Tumors of the Central Nervous System (WHO-2016) but only as a histopathological pattern in the commentary on ganglion cell tumors, but the cIMPACT-6-Utrecht meeting opined that this lesion should be considered a distinct tumor type and could be placed within the section covering "Neuronal and mixed neuronal-glial tumors" as WHO grade I lesion [2-4].

MVNTs usually appear on brain MR imaging (MRI) like a cluster of variably sized nodular lesions (bubbles appearance) located predominantly in the subcortical white matter following the gyral contour. They are characteristically hyperintense on SWI and T2WI,

*Address for Correspondence: Dr. Eduardo De-Oliveira Narvaez, Department of Neuroradiology, BP Medicina Diagnóstica, Hospital Beneficência Portuguesa. R. Maestro Cardim, 769 - Bela Vista, São Paulo - SP, 01323-001, Brazil, E-mail: eonradiologia@gmail.com

Copyright: ( 2020 Narvaez ED, et al. This is an open-access article distributed under the terms of the Creative Commons Attribution License, which permits unrestricted use, distribution, and reproduction in any medium, provided the original author and source are credited. do not suppress on FLAIR or show restricted diffusion and typically do not enhance or cause significative mass effect [2-5]. Usually clinical presentations range from asymptomatic lesion, but refractory seizures, vertigo and dysarthria have been previously described. Commonly these lesions are located in parietal or frontal lobe [2]. We reported an unusual case of MVNTs in deep brain of a half-aged woman with secondary transaxonal degeneration of the limbic system, first diagnosed in 2015, without significant change in five years follow up, and we discuss its morphologic, hemodynamic, diffusion and metabolic properties.

\section{Case Report}

In September 2015, a 55-year-old woman underwent brain MRI in our service for evaluation of seizures. Conventional MRI images shows an infiltrative lesion localized in deep brain of the left cerebral hemisphere, compromising the ventral posteromedial and pulvinar surfaces of thalamus, lateral geniculate body, choroidal fissure, mammillothalamic tract, fornix, septum pellucidum and anterior commissure extending to contralateral globus pallidus and internal capsule, showing hyperintense on T2WI/FLAIR (Figures 1 and 2). High signal was also seen on SWI and DWI, with high ADC values due to the T2-shinethrough effect (Figure 1). No abnormal enhancement was depicted. There was also seen mamillar body atrophy and tapering fornix at left side compatible with degeneration of the mammillothalamic tract, corroborating long term growing of lesion. 


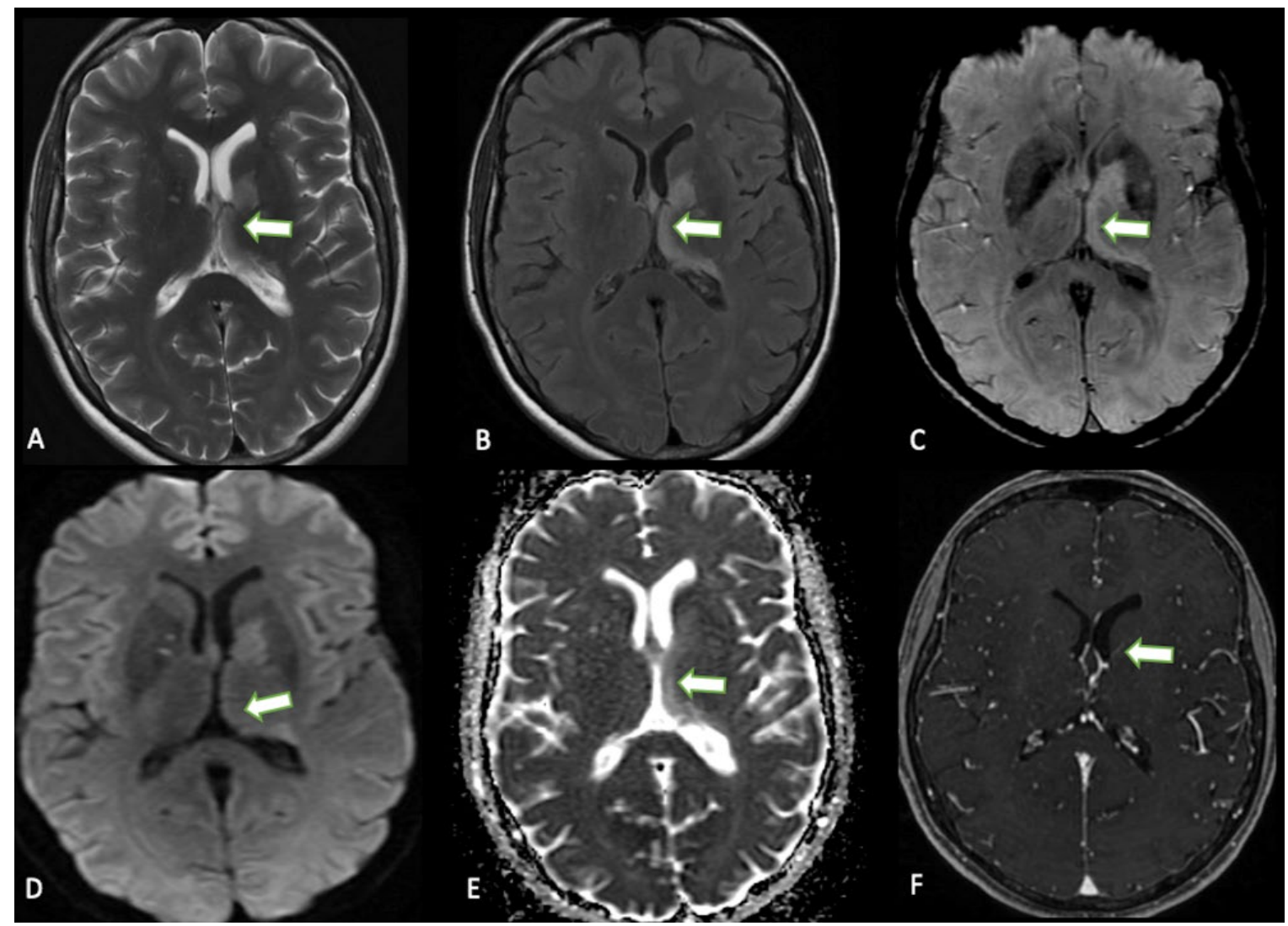

Figure 1. Conventional Axial MRI images shows an infiltrative lesion (arrows) with high signal sign intensity on T2WI and FLAIR (A and B) in depth brain on the left cerebral hemisphere, compromising the ventral, posteromedial and pulvinar surfaces of thalamus, lateral geniculate body, fornix and septum pellucidum. High signal was also seen on SWI and DWI (C and D), with high ADC values due to the T2-shinethrough effect (E). In T1WI post-gadolinium no abnormal enhancement was depicted (F).

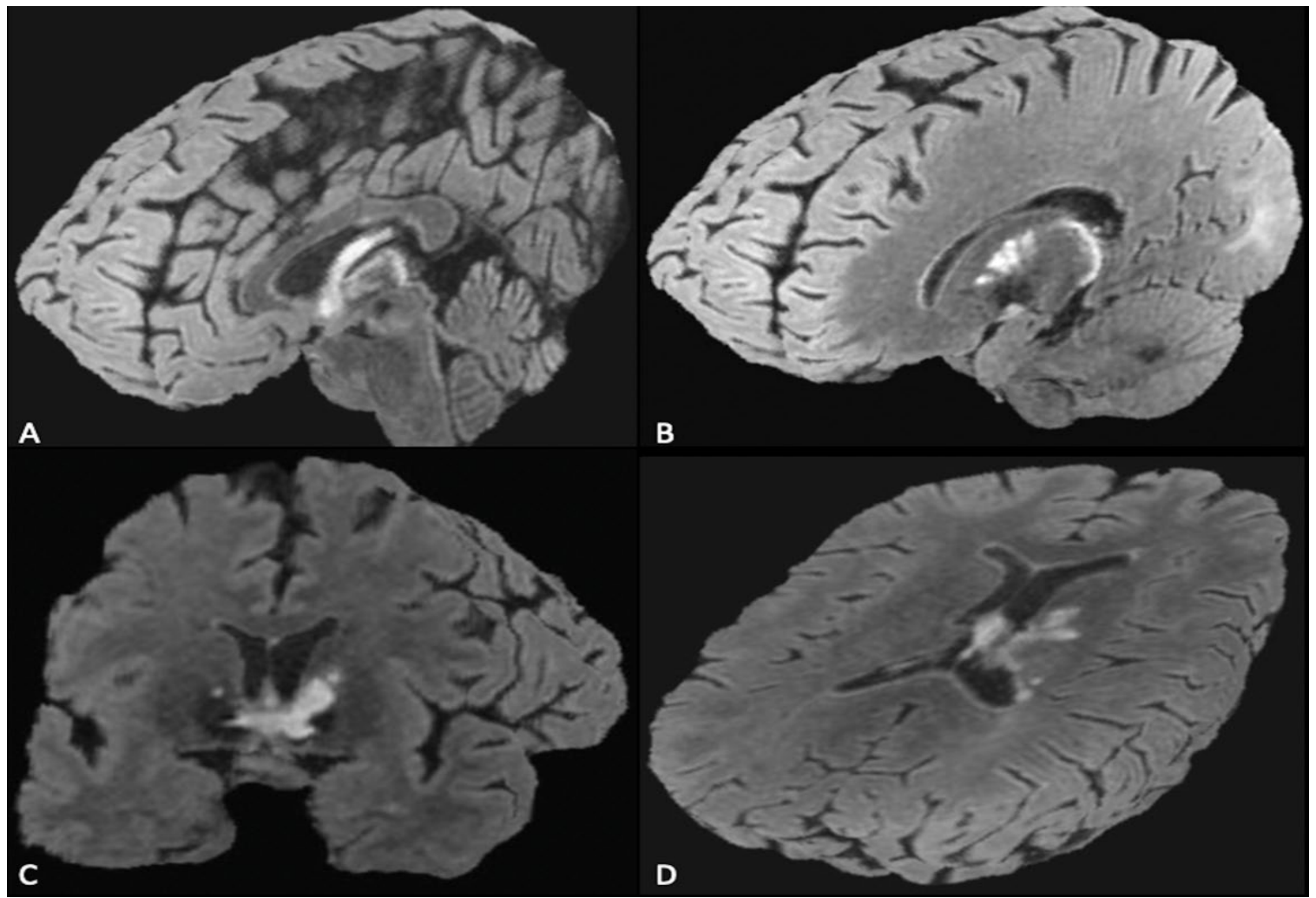

Figure 2. 3D-Flair reconstruction model in Sagital (A and B), Coronal (C) and Axial (D) for evaluation of the bilateral deep cerebral brain, showing infiltrative lesion in the fornix, septum pellucidum, choroidal fissure, thalamus and anterior commissure crossing the midline, with lots of small and confluent nodules in the adjacent white matter.

Dynamic susceptibility contrast-enhanced MR perfusion and Arterial Spin Labeling (ASL) technique were normal (Figure 3). MR spectroscopy demonstrated a mild increase in Choline (Cho) and mild reduction in $\mathrm{N}$-acetyl aspartate (NAA) (Figure 4). Follow up scans was 


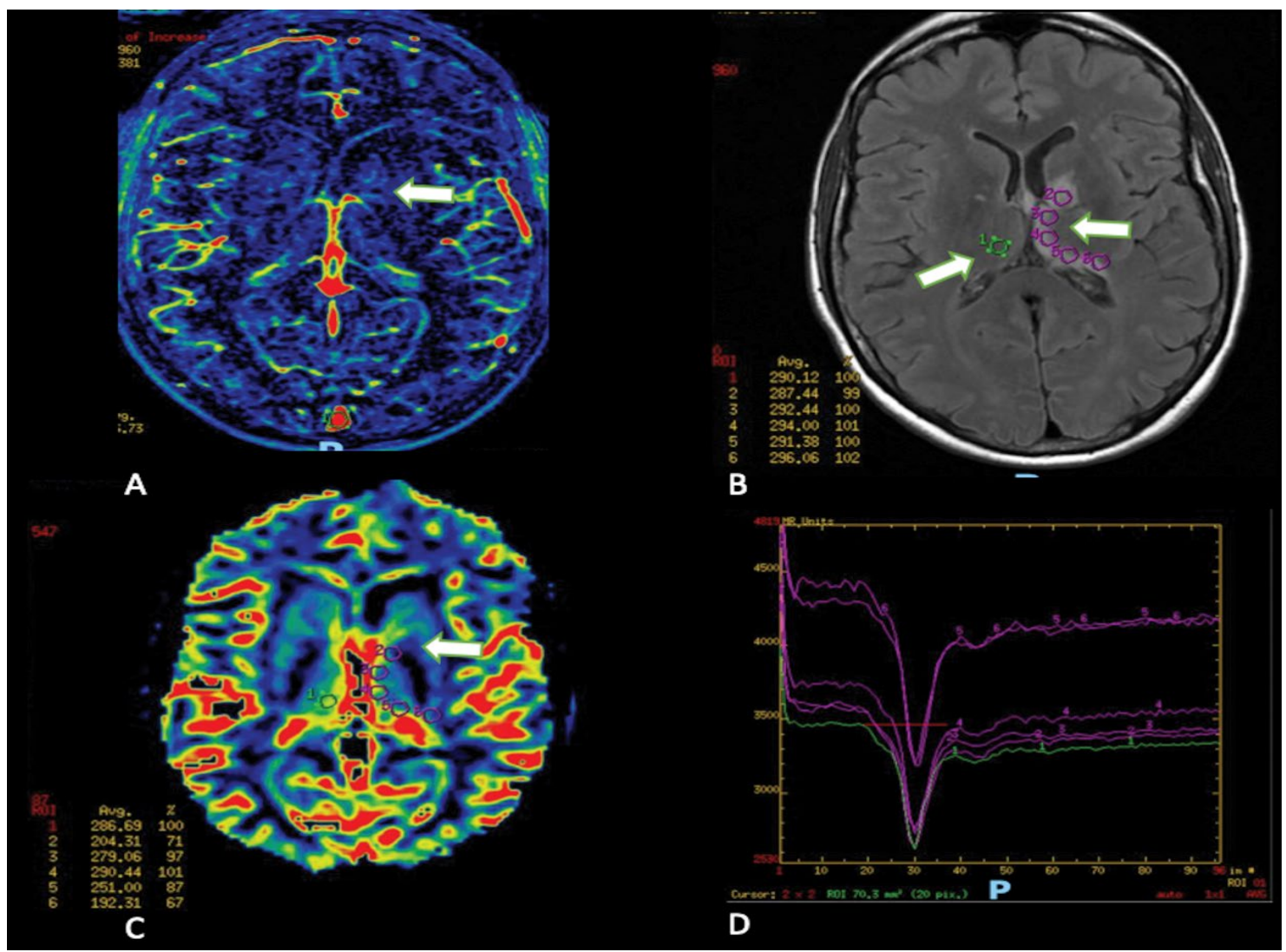

Figure 3. T1-Weighted dynamic contrast-enhanced MR perfusion - DCE-MRI (A) and Dynamic susceptibility contrast (DSC) MR perfusion - CBV values (B, C and D) inside the lesion and in the contralateral white matter with normal appearance (B) (arrows), demonstrates areas with no increase in capillary density and permeability - hypoperfusion (cold perfusion).

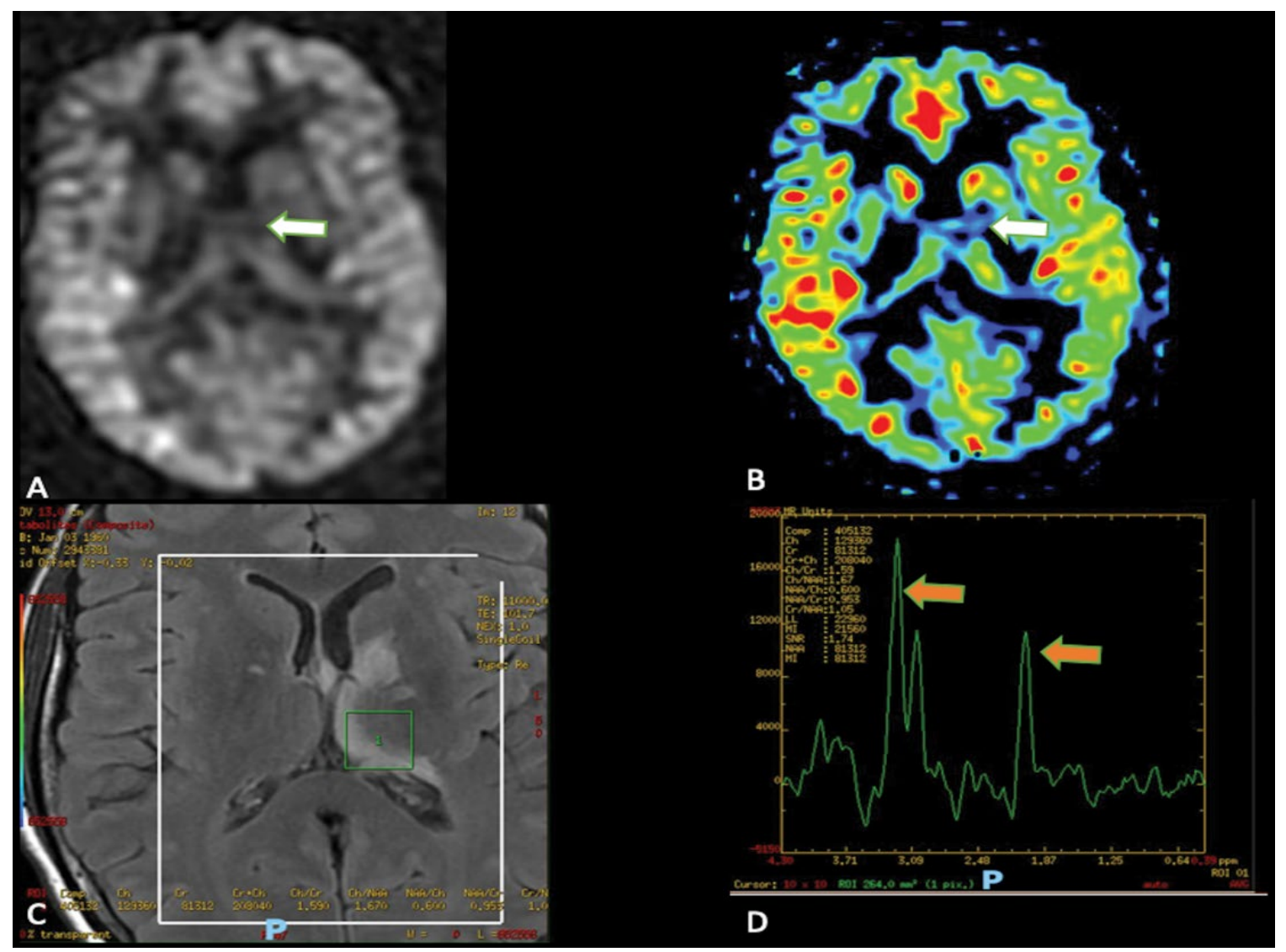

Figure 4. ASL sequences without contrast ( $A$ and $B$ ) shows no $C B F$ values alteration - cold perfusion (white arrows). MR spectroscopy ( $C$ and $D$ ) shows mild increase of choline/creatine ratio (1.59) and mild decrease in NAA values (orange arrows). 
performed for five years and the lesion presented clinically indolent and unchanged in size or appearance.

\section{Discussion}

MVNTs have been recently recognized and show some typical imaging characteristic and most of them are asymptomatic and incidental findings, occurring in adults older than 35 years. Nunes et al reported a case series of 33 patients ages ranged from 8 to 63 years with supratentorial lesions located on the inner surface of an otherwise normal-appearing cortex, commonly in parietal and frontal lobe and without demonstrating interval change [2].

Histologically, MVNTs lesions originates from ganglion cells, which normally present dysplastic and vacuolated, oriented perpendicular to the cortical surface $[2,4]$. Constituent neurons are most often of intermediate-to-large size and are randomly disposed or aligned along thin-walled blood vessels (perivascular arrangement) [4]. Some MVNTs also contain small oligodendrocyte-like elements of unclear lineage. Hypomyelination of the lesions may be observed and the adjacent brain parenchyma appears normal or astrogliotic. By immunohistochemistry, neuronal components are GFAP-negative, consistently express both the $\mathrm{HuC} / \mathrm{HuD}$ neuron-associated antigens and OLIG2, and often display cytoplasmic synaptophysin. Mitotic activity is essentially absent, Ki-67 labeling indices do not exceed $1 \%$ in most cases $[6,7]$.

Multinodular and Vacuolating Neuronal Tumor (MVNT) has been included in the last World Health Organization Classification of Tumors of the Central Nervous System (WHO - 2016) as a unique cytoarchitectural pattern of gangliocytoma [4]. Recent studies have discovered specific immunomarkers that classifies these lesions as a new neoplasm group. MVNTs have been found negative for KIAA1549BRAF fusions and abnormalities involving IDH1 or IDH2, ATRX, TP53, TERT, CIC, FUBP1, PRKCA, CDKN2A, and FGFR1, but MVNTs have been shown to harbor clonal MAPK pathway-activating genetic abnormalities including MAP2K1 exon 2 mutations, non-V600E BRAF mutations and FGFR2 fusions. It has been proposed that they should now be considered as a distinct tumor type and that it could be placed within the section covering "Neuronal and mixed neuronal-glial tumors" as a WHO grade I lesion. This opinion is supported by the observation that MVNTs harbor clonal genetic alterations, which favors a neoplastic rather than malformative origin $[6,7]$.

Conventional MRI is the primary imaging modality for the diagnosis of MVNT and reveals highly characteristic imaging features. CT scans are usually normal without evidence of calcification or mass effect. MR scans shows a unique pattern of multiple, variably sized (usually small) discrete and coalescent nodules along the inner cortical ribbon and subcortical white matter. The nodules are isointense with gray matter on T1WI, hyperintense on T2/FLAIR and on T2 gradient-echo/SWI. Gadolinium enhancement, restricted diffusion, diffuse infiltration or mass effects are typically absent [1-7].

Deep cerebral involvements in MNVTs are not common. Until last year, MVNT in the deep brain has not been found in the literature. This year, Turner et al described MNVT in a 5-years-old boy involving the right basal ganglia and posterior limb of right internal capsule, extending inferiorly to the anterior commissure and right hippocampus [8].

In our case, we present a presumed MVNT that exhibit a remarkably rare similar pattern of imaging findings within an extremally rare location, involving the deep white matter, bilateral basal ganglia, fornix, pellucid septum, corpus callosum, thalamus extending to anterior commissure, with left mammillary body atrophy and ipsilateral tapering fornix due to left transaxonal degeneration of limbic system [9]. Lesion showed increased diffusivity, that suggests lack of mitotic activity and diminished cellularity of the nodules compared to adjacent white matter. Perfusion imaging including was normal and MR spectroscopy showed mild increase of Cho and mild reduction of NAA, in concordance with other studies [10]. We followed for 5 years this presumed MVNTs that exhibit a remarkably similar pattern of imaging findings without any interval change.

\section{Conclusion}

The extremally benign biological behavior of these tumors and the typical imaging features may be sufficient for the diagnosis of this condition, not requiring histological confirmation by biopsy. Therefore, the clinical and imaging stability combined with the deep localization of the lesion observed in our patient were enough to presume the final diagnosis, not requiring the histological examination.

\section{References}

1. Huse JT, Edgar M, Halliday J, Mikolaenko I, Lavi E et al. "Multinodular and Vacuolating Neuronal Tumors of the Cerebrum: 10 Cases of a Distinctive SeizureAssociated Lesion". Brain Pathol 23 (2013): 515-524.

2. Nunes RH, Hsu CC, da Rocha AJ, Amaral LLF, Godoy LFS et al. "Multinodular and Vacuolating Neuronal Tumor of the Cerebrum: A New “Leave Me Alone" Lesion with a Characteristic Imaging Pattern". Am J Neuroradiol 38 (2017): 1899-1904.

3. Louis DN, Perry A, Reifenberger G, von Deimling, Figarella-Branger D et al. "The 2016 World Health Organization Classification of Tumors of the Central Nervous System: A Summary". Acta Neuropathol 131 (2016): 803-820.

4. Makrakis D, Veneris S, Papadaki E. "Multinodular and Vacuolating Neuronal Tumor Incidentally Discovered in a Young Man: Conventional and Advanced MRI Features". Radiol Case Rep 13 (2018): 960-964.

5. Aira SO, Velasco MR, Ronco MS et al. "Multinodular and Vacuolating Brain Tumors; What Radiologist Needs to Know". Poster ECR.

6. M Pekmezci, M Stevers, JJ Phillips, J Van Ziffle, BC Bastian, NM Tsankova "Multinodular and Vacuolating Neuronal Tumor of the Cerebrum is a Clonal Neoplasm Defined by Genetic Alterations that Activate the MAP Kinase Signaling Pathway". Acta Neuropathol 135 (2018): 485-488.

7. Louis DN, Wesseling P, Aldape K, Brat DJ, Capper D et al. "Cimpact-NOW Update 6: New Entity and Diagnostic Principle Recommendations of the Cimpact-Utrecht Meeting on Future CNS Tumor Classification and Grading". Brain Pathol 30 (2020): 844-856.

8. Turner AL, D'Souza P, Belirgen M, AL-Rahawan MM. “Atypical Presentation of Multinodular and Vacuolating Neuronal Tumor of the Cerebrum in A Boy". $J$ Neurosci Rural Pract 11 (2020): 214-215.

9. Kinoshita F, Kinoshita T, Toyoshima H, Shinohara Y. "Ipsilateral Atrophy of the Mammillary Body and Fornix after Thalamic Stroke: Evaluation by MRI". Acta Radiol 60 (2019): 1512-1522.

10. Badat N, Savatovsky J, Charbonneaue F, Collin A, Lecler A. "Multinodular Vacuolating and Neuronal Tumor of the Cerebrum". Neurol 89 (2017): 304-305.

How to cite this article: Narvaez EO, Marussi VHR, Junior CLS and Hatano NT, et al. "Multinodular and Vacuolating Neuronal Tumor in the Brain Depth - Atypical Presentation of a New Tumor: A Case Report." J Mol Genet Med 14 (2020): 453 doi: 10.37421/jmgm.2020.14.453 